\title{
EL CUIDADOR DEL ADULTO MAYOR: MIRADA EN TIEMPOS DE COVID-19
} THE BIGGEST ADULT'S CARETAKER: LOOK IN TIMES DE COVID-19

\author{
Dra. C. Celín Pérez Nájera ${ }^{l}$ \\ celinpn1973@gmail.com \\ Lic. Lisset Díaz Echemendia ${ }^{2}$ \\ Dra. C. Xiomara Cabrera Cabrera ${ }^{3}$ \\ Lic. Bertha Rosana Llontop Castañeda ${ }^{4}$ \\ Dr. C. Juan Carlos Callejas Torres. ${ }^{5}$
}

Cuba/Perú

\section{SUMARIO}

- Introducción

- Concepciones doctrinales del cuidador

- Clasificaciones del cuidador del adulto mayor

- La atención del Estado a los adultos mayores ante la introducción y propagación de la COVID-19: mirada desde Cuba

- Elementos configurativos de la figura del cuidador del adulto mayor

- Protección jurídica en Cuba al cuidador del adulto mayor

- Conclusiones

- Bibliografía.

\section{RESUMEN}

La figura del cuidador del adulto mayor es una de las líneas de investigación del proyecto "Violencia en la ancianidad" que asume como objetivo proporcionar un material bibliográfico actualizado sobre el tema desde las ópticas criminológica y victimocriminológica. Con énfasis en el cuidado de los gerontes en Cuba y su proyección para enfrentar la pandemia del coronavirus. La metódica investigativa proporcionó una orientación dialéctico-materialista del entorno, que facilitó la investigación mediante los métodos: histórico-lógico, analítico-sintético, inductivodeductivo, teórico- jurídico y sociológico. Concibiendo como resultados esenciales una fundamentación teórica desde la Criminología sobre la conceptualización de esta figura, la determinación de los elementos configurativos del cuidador del adulto mayor, valoramos también la atención del Estado a este grupo etario ante la introducción y propagación de la COVID-19 con una mirada desde Cuba y dentro de ello proponemos juicios organizativos de sus variantes en que puede presentarse, así como el análisis de su regulación jurídica en las legislaciones nacionales.

\section{ABSTRACT}

The figure of the biggest adult's caretaker is one of the lines of investigation of the project "Violence in the old age" that assumes as objective to provide an up-to-date

1 Doctora en Ciencias Jurídicas y Especialista en Derecho Penal. Profesora Titular de la Facultad de Ciencias Sociales y Humanísticas. Rectora de la Universidad de Ciego de Ávila Máximo Gómez Báez. Cuba. https://orcid.org/0000-0002-8964-6427

2 Licenciada en Derecho. Abogada de la Organización de Bufetes Colectivos del municipio de Ciego de Ávila. Provincia de Ciego de Ávila. Cuba. E-mail: lisset.dias@cav.onbc.cu https://orcid.org/0000-0002-6058-762X

3 Doctora en Ciencias Pedagógicas, Especialista en Derecho Penal. Docente de Investigación en la Escuela de posgrado Universidad Señor de Sipán, Perú. E-mail: xiomaracabreracabrera@gmail.com https://orcid.org/0000-0002-4783-0277

4 Licenciada en Marketing y Dirección de Empresas. Maestría en Administración de Negocios. Jefa de Relaciones Internacionales de la Universidad Cesar Vallejo en Chiclayo. E-mail: rosanillontop@gmail.com https://orcid.org/0000-0002-1209-0002

5 Doctor en Aportaciones Educativas en Ciencias Sociales y Humanas y Doctorado en Ciencias de la Educación. Docente de Investigación en la Escuela de Posgrado Universidad Señor de Sipán, Perú. E-mail: callejas1959@yahoo.es https://orcid. org/0000-0001-8919-1322 
bibliographical material on the topic from the optic criminological and crime victim. With emphasis in the care of the gerontes in Cuba and their projection to face the pandemic of the Coronavirus. The Methodical Investigative it provided us a dialectical-materialistic orientation of the environment that facilitated the investigation by means of the methods: historical-logical, analytic-synthetic, inductive-deductive, theoretical - juridical and sociological. Conceiving as essential results a theoretical foundation from the Criminology on the conceptualization of this figure, the determination of the elements configurative of the biggest adult's caretaker, we also value the attention from the State to this group ethereal before the introduction and propagation of the COVID-19 with a look from Cuba and inside we propose it organizational trials of the variants in that it can be presented, as well as the analysis of their artificial regulation in the national legislations.

\section{PALABRAS CLAVES}

Adulto mayor, cuidador, envejecimiento, pandemia, vulnerable.

\section{KEY WORDS}

Bigger adult, caretaker, aging, pandemic, vulnerable.

\section{INTRODUCCIÓN}

La Criminología, como acervo de teorías y conocimientos de la realidad, presenta un abanico de posibilidades que incorpora a la estructura social y se desarrolla como parte integrante de un proceso científico único, (Navarrete, 2004, p. 8) para ello demanda de la oportuna formación de conocimientos en el análisis de los problemas sociales complejos, los que requieren un proceso de construcción estrechamente vinculados entre sí, a partir de la cooperación en equipos y comunidades de científicos, profesionales y técnicos de diversos campos; de ahí la necesidad de incursionar en el proceso del envejecimiento poblacional como fenómeno social.

Este envejecimiento afecta a la población a escala mundial, aunque a diferentes ritmos, o sea, unos más acelerados que otros, pero de un modo inexorable se producirá la transición demográfica, lo que obliga a la familia, el Estado y la estructura política, económica a abordar la figura del geronte como un fenómeno sin precedente que requiere un enfrentamiento multisectorial, con una visión interdisciplinar, diversificada, especializada e integral.

La población mundial continúa creciendo de una manera alarmante, en la actualidad somos 7,000 millones de personas y para el 2050 se pronostica la cifra de 9,500 millones de habitantes. Preocupa entonces el análisis de las estadísticas en los adultos mayores, en las que se predice que el número de personas mayores de 60 años en el planeta se triplique, de 605 millones a casi 2,000 millones en el 2050. Cuba, con un $20.8 \%$ de su población mayor de 60 años, es uno de los países más envejecidos de América Latina y está previsto sea en el 2030 uno de los más envejecidos del mundo con el $30.1 \%$ de la población". (Pérez, 2012, p. 2; Organización Mundial de la Salud, 2018, p. 2; Oficina Nacional de Estadísticas e Información, 2018, p. 12).

En este caso,

[...] el envejecimiento poblacional se produce por la acción combinada de dos connotadas transformaciones sociales que poseen una fuerte relación entre sí: la transformación demográfica y la transformación epidemiológica o de salud. Definidas brevemente, la transformación demográfica se refiere a los cambios en las estructuras de la población, debido esencialmente a las bajas tasas de fecundidad y de mortalidad existentes en nuestro país. La transformación epidemiológica responde a los enormes avances en materia de salud humana, progresos caracterizados por la existencia de menor incidencia, prevalencia y letalidad de las enfermedades crónicas degenerativas e incapacitantes. (Pérez, 2012, p. 3).

Para la investigación se utilizaron los métodos histórico-lógico, analítico-sintético, inductivodeductivo, teórico- jurídico y sociológico. Estas valoraciones generalizadoras y sistematizadoras tienen un enfoque modelativo y sistémico-estructural.

Cuando analizamos los fenómenos y procesos que se producen en la sociedad el más complejo de todos los sistemas existentes, es necesario considerar los rasgos generales de la causalidad y lo específico de la vida social. Lo específico indica que todas las regularidades sociales se realizan a través de la actividad de las personas. Se puede por tanto relacionar 
una serie de particularidades de los vínculos causales en el medio social, (Navarrete, 2004 , p. 21) desde esa óptica criminológica reviste una especial importancia el estudio del contexto social y del grupo de riesgo, debido a sus propias características de sensibilidad y fragilidad por la edad, potenciando desde una visión victimocriminológica su estudio y atención a partir de ser considerado un grupo victimal de la realidad social.

Es durante esta etapa de la vida donde el ser humano se torna más vulnerable y necesitado de cuidados, hecho que nos obliga a enfrentar con más premura la situación actual de los cuidadores en el tratamiento al adulto mayor, nos encontramos viviendo una de las realidades más difíciles y con alto costo humano que ha tenido la historia, la pandemia de enfermedad por coronavirus que se identificó por primera vez en diciembre de 2019 en Wuhan, al presentarse un grupo de personas con neumonía de etiología desconocida, su nivel de expansión y afectación en el mundo ha sido inimaginable, se han informado 3'575,412 contagios en más de 184 países con más de 253,717 muertes lamentables y 1'181,403 personas recuperadas. Cuba no ha quedado exenta se muestra una cifra de 1,685 pacientes confirmados con 69 fallecimientos y 954 recuperados. (OMS, 2018, p. 3; Ministerio de Salud Pública de Cuba, 2020, párrafo 6).

A estas alturas, ya nadie ignora que la pandemia no es sólo una crisis sanitaria. Es lo que las ciencias sociales califican de "hecho social total", en el sentido de que convulsa el conjunto de las relaciones sociales, y conmociona a la totalidad de los actores, instituciones y valores. La humanidad está viviendo con miedo, sufrimiento y perplejidad una experiencia inaugural. (...) Nadie sabe interpretar y clarificar este extraño momento de tanta opacidad. (...) Angustiados, los ciudadanos vuelven sus ojos hacia la ciencia y los científicos, como antaño hacia la religión, implorando el descubrimiento de una vacuna salvadora, (...) se busca también refugio y protección en el Estado. (Ramonet, 2020, p. 12).

Las personas adultas mayores,

[...] plantean desafíos específicos, al constituirse como una población heterogénea en términos de salud, discapacidad y demanda de servicios, lo cual insta y reclama atención priorizada y especializada desde toda la sociedad. Debemos tener en cuenta que, junto al progresivo proceso de envejecimiento, aumentan los índices de dependencia de estas personas, por ello, las salidas a este problema demográfico tan importante transitan por el desarrollo atemperado y evolutivo de las estructuras sociales, económicas y culturales. (Pérez, 2012, p. 2).

De ahí, la necesidad de su atención y proyección en el cuidado ya sea por la familia o el Estado; trazando adecuadas políticas que respalden el proceso de envejecimiento y velen por la multidisciplinariedad de sus acciones; las que, sin lugar a dudas, muestran una visión de futuro con carácter humanista.

\section{CONCEPCIONES DOCTRINALES DEL CUIDADOR}

Para abordar la figura del cuidador analizamos su carácter multidimensional, el cual comprende un conjunto de elementos integradores de diferentes dimensiones centradas en la atención a la persona, las que están relacionadas con las características sociales, familiares y culturales relativas al bienestar de los individuos vulnerables.

Debemos partir de evaluar la distinción entre los términos cuidar y asistir, con el objetivo de comprender con más facilidad los conceptos que se expresan. "Asistir y cuidar pueden ser sinónimos, sin embargo, el primero significa acompañar, servir y socorrer; en cambio el segundo, se refiere a los criterios de atender, esmerarse y mimar". (Alvero, 1978, p. 97 y p. 215).

"En la práctica la diferencia pasa por la palabra amor, el que asiste sirve, el que cuida sirve con amor, ternura dedicación y pasión, una madre no asiste a su hijo, una madre cuida a su hijo". (Pérez, 2016, p. 2). El cuidar implica atención personal, vigilancia y acompañamiento, brindar cariño, entrega, comprensión, apoyo y ayuda en las labores que resultan ser un poco complejas para la persona necesitada, es hacerse cargo de una persona y velar por su bienestar.

Se define, además,

[...] como una relación y un proceso; no es la mera ejecución de tareas o la realización de procedimientos prescritos por un médico, el objetivo de los cuidados va más allá de la enfermedad, es una clase de relación constituida 
por una disposición genuina para con el otro, reciprocidad y el compromiso de promover el bienestar del otro. El cuidado es un trabajo de amor y con ello se manifiesta su dualidad: el amor o interés emocional por la persona que recibe los cuidados y el aspecto práctico de cuidar a otro. (De la Cuesta, 2013, p. 34).

Por su parte, se destaca que,

[...] el cuidar surge con la propia aparición humana, y presenta dos formas de significado, por una parte, el cuidado consigo mismo y por otra, el cuidado con el otro; define un tipo de relación social específica, basada en afecto y servicio, cariño y trabajo, que se realiza a través de la capacidad de cuidado informal de mujeres, madres, hijas, vecindad o amistad, o de cuidado formal prevista por enfermeras, maestras, trabajadoras sociales, entre otras, (Guimaráes, 2015, p. 86).

Esta es la conceptualización que acogemos en nuestra investigación para abordar a la persona destinada al cuidado de los gerontes.

El cuidador es en no pocas ocasiones el recurso, instrumento y medio por el cual se proveen cuidados específicos y muchas veces especializados a los enfermos crónicos, es decir, en ellos se deposita o descansa el compromiso de preservar la vida de otro". Se define también como "aquella persona que asiste o cuida a otra afectada de cualquier tipo de discapacidad, minusvalía o incapacidad, que le dificulta o impide el desarrollo normal de sus actividades vitales o de sus relaciones sociales. (Cruz Rivas, 2016, p. 3; De los Reyes, 2008, p. 12).

Proporcionar asistencia es una forma de que las personas desprovistas sientan que sus necesidades físicas, sociales y afectivas están solucionadas, para los cuidadores, implica una consagración, que conlleva esfuerzos no solos propios sino del resto de la familia.

Cuando analizamos los conceptos encontramos dos enfoques esenciales, en un primer momento una relación-proceso, es decir compromiso y reciprocidad del cuidador y quien se cuida; y una segunda variante mediante un rol social concretada en la capacidad de cuidado informal que posee; en ambos casos existe una misma perspectiva de valoración, la correlación tiene que estar establecida en afecto, responsabilidad y protección.

\section{CLASIFICACIONES DEL CUIDADOR DEL ADULTO MAYOR}

La figura del cuidador del adulto mayor ha sido abordada desde disímiles entornos, tales como: la medicina, lo social o lo jurídico; en cada uno de ellos encontramos una amplia gama de clasificaciones el cuidador formal, el informal, los principales o primarios, los secundarios, los empíricos, profesionales, los domiciliarios, así como por beneficios. Al analizar la variedad de prototipos de cuidadores del geronte y en aras de conformar una agrupación clasificatoria concebimos dos grandes grupos de cuidadores: formales e informales.

\section{Los cuidadores formales,}

[...] están provistos por grupos u organizaciones que no están unidos por lazos de parentesco o amistad con la persona que recibe los cuidados, estos cuidadores reciben un beneficio patrimonial por la acción de cuidar y son ofrecidos en instituciones. Dentro de ellos contamos con los cuidadores empíricos y profesionales. (Díaz, 2016, p. 16).

Los cuidadores informales, "se presentan con una amplia clasificación, se caracterizan por rasgos esenciales, que son los que en general lo identifican", (Díaz, 2016, p. 17) este tipo de cuidados se concibe como no institucional, puede ser remunerado o no, provisto generalmente por una relación de parentesco. Está constituido por " cuidadores independientes, parte de la familia que trabajan por su cuenta, para cuidar o buscan en esa vía la utilización de un empleo, este se considera constituido por las actividades de provisión de servicios personales en el ámbito doméstico a sujetos con necesidades especiales". (Gallardo y Rojas, 2016, p. 17). Dentro de ellos encontramos los domiciliarios, los primarios, secundarios, empírico y por beneficio.

Según estas clasificaciones generalmente los cuidadores que prestan la ayuda informal son los hijos, cónyuges y nueras, tal y como analizaremos más adelante.

Resumiendo lo anterior clasificamos la figura del cuidador desde dos ópticas diferentes, el cuidado informal fundado en una correlación social basada en sentimientos afectivos que se realiza a través de una relación de vínculo 
parental o afecto y el cuidado formal ejecutado por enfermeras, personal institucionalizado, entre otras.

\section{LA ATENCIÓN DEL ESTADO A LOS ADULTOS MAYORES ANTE LA INTRODUCCIÓN Y PROPAGACIÓN DE LA COVID-19: MIRADA DESDE CUBA}

La impaciencia que se vive en el mundo,

[...] nos obliga a una mirada diferente a las sociedades desde la política, economía, los sistemas de salud, las estructuras sociales, la desprotección de los grupos más vulnerables, son sucesos que deben servir de experiencia para reformular el contrato social y avanzar hacia más altos niveles de solidaridad comunitaria y mayor integración social. Cualquier respuesta postpandémica debería apoyarse en los principios de una economía verdaderamente regenerativa, basada en el cuidado y la reparación. (Ramonet, 2020, p. 12).

Indudablemente defendemos un modelo global más humano y solidario.

Se ha demostrado que los gerontes poseen más posibilidades de agravarse al contraer COVID-19, esencialmente porque según se envejece, el sistema inmunológico va cambiando, dificultándose el enfrentamiento ante enfermedades e infecciones y tienen más probabilidades de presentar problemas de salud subyacentes, lo cual agrava su recuperación. Con la edad se aumenta el riesgo que el sistema respiratorio o los pulmones se cierren.

En la cotidianidad la desconfianza e incertidumbre han crecido. Algunos fanáticos ultraliberales no han tardado en reclamar sin tapujos la eliminación maltusiana de los más débiles. El vicegobernador de Estados Unidos Dan Patrick declaró que los abuelos deberían sacrificarse y dejarse morir para salvar la economía. En Holanda, el Primer ministro ultraliberal Mark Rutte, apuesta por la inmunidad de rebaño, el jefe de epidemiología del Centro Médico de la Universidad de Leiden, Frits Rosendaal, declaró que no se deben admitir en las unidades de cuidados intensivos a personas demasiado viejas. La COVID-19 no distingue, pero las sociedades desigualitarias sí, (Ramonet, 2020, p. 12).

Al escuchar estos criterios, se pueden evaluar cuáles son las medidas de atención y protección a este grupo vulnerable.
Para ello debemos contar con,

[...] un sistema de prevención legitimado por la voluntad política y la activa participación de los ciudadanos donde los Estados que no han desarrollado políticas adecuadas para apoyar a la persona y al cuidador, (...), tengan la obligación de garantizarle todos sus derechos y eso incluye darles apoyo para que tengan actividades durante el día, para que puedan tomar sus decisiones y para que puedan seguir participando... Independientemente, están emprendiendo medidas sanitarias para reducir los contagios y de contención social en favor de los grupos más vulnerables para tratar de contrarrestar los efectos económicos negativos de la pandemia. También están tomando medidas económicas, fiscales y monetarias. (Devandas, 2019, párrafo 4; Hadar, 2020, párrafo 6).

No debemos perder de análisis que el efecto positivo que debe existir en el grupo etáreo no se percibe, falta la prevención intencionada estructural, la que conlleva un grupo de acciones multidisciplinarias para integrarlo socialmente.

La sociedad debe ser solidaria y proteger mejor a las personas mayores, uno de los grupos más afectados por la pandemia, las medidas de distanciamiento social no deben convertirse en exclusión. Los protocolos de prueba deben basarse en las necesidades de salud, y deben descalificar los criterios que no sean médicos, como la edad o la discapacidad. (KornfeldMatte, 2020, párrafo 12; Adhanom, 2020, párrafo 7).

La mayoría de los Estados no se prepararon desde sus poderes y estructuras para afrontar esta enfermedad. La pandemia dejó de ser un problema del sector de la Salud para convertirse en un fenómeno global, con esa mirada integradora tienen que afrontar la afectación desde los sectores sociales, económicos $\mathrm{y}$ políticos; evidenciándose la desorganización en los sistemas de salud, máxime cuando las políticas privatizadoras han demostrado los crueles resultados vividos. Otro de los errores más comunes ha sido la falta de vigilancia de las estadísticas mundiales ya sea por subestimar la pandemia o por no crear colocaciones necesarias para su enfrentamiento.

Además de la prevención, necesitamos de la adecuada previsión, todos debemos ser conocedores de estas políticas en materia de prevención de riesgos y comprenderlas para ser capaces de acatarlas de forma inmediata y 
paliar así los riesgos lo más posible”. Mientras mayor desigualdad exista en las estructuras de un país, "los grupos más vulnerables soportarán el peso de las repercusiones económicas de la pandemia y dispondrán de menos recursos para combatirla. Se debe prestar especial atención a las mujeres por su doble función de trabajadoras y cuidadoras. (Fernández, 2020, párrafo 6; Adhanom, 2020, párrafo 9).

Las sociedades van conformando su proceso de desarrollo, esa identidad reconoce criterios definitivos con relación al orden social que se protege, al interés social que prevalece desde la supremacía de los grupos de poder, al proyecto organizativo de su sociedad civil, entre otras. La sociedad cubana exhibe particularidades únicas en su práctica sobre el control al sistema de salud y en tal sentido dedicaremos nuestra valoración.

Entre las potencialidades que propician el beneficioso crecimiento de la calidad en la salud se encuentra el perfeccionamiento de las organizaciones comunitarias, y la práctica interactiva de la sociedad civil en labores sociales, el actual desarrollo cultural y educacional, entre otras. Precisamente la experiencia socio-preventiva cubana y las capacidades de la sociedad permiten revisar la situación de forma integrada.

\section{Cuba cuenta con un,}

Plan Nacional aprobado por el Consejo de Ministros, y dirigido por la máxima autoridad del país, que no solo integra al sistema de Salud Pública, sino a todos los organismos. El plan se basa en la contención al mínimo el riesgo de la introducción y propagación de la COVID-19 en el territorio nacional, así como la disminución de los efectos negativos que trae consigo una epidemia en la salud de la población y su impacto en la esfera económica-social del país. (MSP, 2020, párrafo 11).

Se reorganizaron "los servicios médicos ante la amenaza de la COVID- 19, para evitar la afluencia innecesaria de personas a las instituciones sanitarias y sin poner en riesgo la vida de los habitantes, apostando por las pesquisas activas efectuadas por el personal médico de conjunto con organizaciones de masas". (Portal, 2020, párrafo 3). El sistema de vigilancia está basado fundamentalmente en la organización de la atención primaria de salud.
Unido a ello se desarrolla un proceso de preparación de todo el personal de salud, para que este sea capaz de evaluar a cada paciente e identificar los casos que puedan ser sospechosos de esta enfermedad. Se organizó un grupo de especialistas de los centros de investigaciones con el objetivo de diseñar y ejecutar tareas para el desarrollo de nuevos conocimientos, productos y servicios relacionados con la COVID-19.

La dirección del país ha decidido los centros y hospitales que serán utilizados territorialmente, para el aislamiento y proceder de contactos y sospechosos de la enfermedad, en este caso las universidades se encuentran siendo utilizadas en su rol social como centros de aislamientos, como parte del flujo de pacientes, desde su identificación como sospechoso, el tránsito en la institución designada; así como los protocolos de tratamiento a seguir.

Se desarrolla un "sistema de capacitación a profesionales, trabajadores y estudiantes del sector Salud, así como de otros Organismos de la Administración Central del Estado, organizaciones y trabajadores por cuenta propia vinculados al turismo". (MSP, 2020, párrafo 8). Los cursos se apoyan en materiales docentes contextualizados al ámbito de desempeño o labor de cada trabajador, para elevar el nivel de la capacitación. El proceso consta de varias etapas para garantizar la participación directa en la comunidad. Se cuenta además con un Plan de comunicación, con un fin comunicativo y preventivo.

Aunque el sistema de salud cubano presenta carencias e irregularidades, continúa apostando por el desarrollo técnico y profesional, con el fin de continuar defendiendo la calidad de vida de toda la población, esencialmente desde la prevención.

Esta isla, subdesarrollada, bloqueada, ha acondicionado lugares para cuarentena; ha desplegado un pesquisaje a toda la población con énfasis en los adultos mayores; produce los medicamentos necesarios para su tratamiento; ha enviado misiones médicas a varios países para enfrentar el virus. (Anaya, 2020, párrafo 6). Continúa defendiendo que lo más importante es la vida humana. 


\section{EL CUIDADO DE LOS ADULTOS MAYORES FRENTE A LA PANDEMIA COVID-19}

La Gerontología muestra una visión integral del desarrollo en el período final del ciclo vital humano, describe a los adultos mayores como sujetos en pleno desarrollo psicológico, con potencialidades para el aprendizaje y para la diversificación de sus actividades cotidianas. Esta visión de la capacidad de desarrollo potencial debe primar en la labor de las personas que se encargan de la atención, cuidado y esparcimiento de los gerontes, en el nivel familiar o institucional, personas a las que denominamos cuidadores. (Pérez, 2012, p. 57).

Esta figura abarca variadas representaciones $\mathrm{y}$ en consecuencia demanda de respuestas interdisciplinarias; se caracteriza por la multiplicidad de formas de presentarse en la sociedad.

La COVID-19 ha obligado al planeta a un confinamiento social no esperado, y para los adultos mayores la realidad ha sido más impactante cuando han sido identificados como uno de los grupos de riesgos más sensible, teniendo que acudir al cuidador, es por ello que proponemos varios juicios organizativos donde coincide con el ambiente o contexto espacial de ocurrencia:

- Cuidadores para adultos mayores, que viven en el seno de la familia.

- Cuidadores para adultos mayores a cargo del Estado. (Hogar de ancianos o casas de abuelos).

- Cuidadores para adultos mayores que viven solos, poseen su asistencia o seguridad social.

- Adultos mayores desprotegidos totalmente.

Para esclarecernos conceptualmente examinaremos las variantes clasificatorias mencionadas.

\section{Cuidadores para adultos mayores, que viven en el seno de la familia}

Los cuidadores familiares son esenciales en el cuidado y bienestar de los adultos mayores, resultan la solución ideal para las familias durante esta crisis sanitaria, en la mayoría de los casos se esmeran en asistir y proteger a sus seres queridos de cualquier riesgo que pueda enfermarlos. Se encargan de atenderlos para que los otros miembros de la familia efectúen sus diligencias, el sexo predominante de estas personas que cuidan es el femenino y su conexión parental fluctúa, entre las hijas, las nueras y la esposa o esposo cónyuge.

\section{Cuando,}

[...] la persona que cuida es el hijo, existe un vínculo natural familiar con la persona dependiente que favorece la disposición del cuidado, en la mayoría de las ocasiones, representa un fuerte impacto emocional para los hijos darse cuenta de que el padre, la madre o ambos ya no pueden valerse por sí mismos. (Díaz, 2016, p. 18).

Con esta situación de aislamiento social las medidas de cuidado tienen una creciente demanda lo que conlleva a una labor más intensa de higiene, aseo y alimentación; y por ende un gasto económico no planificado. La mayoría de los cuidadores no logran buscar alternativas laborales, pues las medidas extremas han sido en casi todos los sectores, pero no se relacionan con el exterior por miedo al contagio de ese adulto más vulnerable.

"Los hijos e hijas de los cuidadores deben atender también a las necesidades de su familia y propias, decidir dónde, cuándo y cómo invertir los esfuerzos y energías es difícil, sobre todo cuando la realidad asume esfuerzo y sacrificio". (De la Cuesta, 2013, p. 41).

En el caso de

[...] las nueras cuidadoras, aquí no existe un vínculo consanguíneo con la persona cuidada, solo media una relación de convivencia que no siempre está amparada en sentimientos de cariño o afecto, hacia la persona cuidada. Estas circunstancias pueden derivar en consecuencias negativas en la persona objeto del cuidado, conduciendo a malos tratos, carencia de afecto y confianza por la persona que cuida, quisiéramos precisar que no en todos los casos ocurre de la misma forma, existen nueras que cuidan con tanto amor, y dedicación como el propio hijo o hija. (De la Cuesta, 2013, p. 42).

El otro caso que se presenta son "el esposo o la esposa, cuando uno sufre un deterioro de la salud y necesita ayuda para sus actividades de la vida diaria, el cuidador principal suele ser el miembro de la pareja con mejor salud, la nueva situación que supone cuidar al esposo 
o a la mujer puede resultar difícil y exige una constante adaptación". (De la Cuesta, 2013, p. 43). La comunicación franca y amable ayuda notoriamente en la difícil convivencia llegando a valorarse en oportunidades solo como un compromiso propio del matrimonio.

\section{Cuidadores para adultos mayores a cargo del Estado. (Hogar de ancianos o casas de abuelos)}

"Los cuidadores expertos de instituciones son personas capacitadas a través de cursos teórico-prácticos de formación, dictados por equipos multi e interdisciplinarios de docencia, para brindar atención preventiva, asistencial y educativa al anciano y a su núcleo familiar" (Pérez y Moya, 2000, p. 6) estas se ubican en Cuba en las entidades de Salud Pública.

Las moradas para personas mayores, hogares de ancianos o residencia geriátricas resultan un entorno de gran vulnerabilidad por ser un lugar cerrado donde es difícil evitar el contacto entre los numerosos pacientes, en oportunidades no se encuentran con el personal sanitario requerido. La persona responsable de los cuidados no debe tener factores de riesgo de complicaciones, será considerada contacto estrecho y deberá realizar cuarentena domiciliaria durante 14 días.

En la actualidad hay en

[...] Cuba 155 hogares de ancianos, con una disponibilidad de más de 11,000 camas, y 294 casas de abuelos con régimen seminterno. Para ser atendido en estas últimas es preciso tener 60 años o más, tener autonomía en sus actividades diarias, no poseer deterioro cognitivo avanzado y tener alguna problemática social, como que la familia trabaje y deje a la persona mucho tiempo sola, o haber enviudado en un matrimonio de muchos años, algo que afecta en esas edades de manera especial. (Barrios et al, 2020, párrafo 17).

Este colectivo es también uno de los más proclives a adquirir el virus puesto que tienen necesidad de cuidados o asistencia, una preocupación especial por los que han sido excluidos de la sociedad y viven en la pobreza, con acceso limitado a los servicios de salud o en espacios de confinamiento como prisiones o asilos. La distancia social no debe convertirse en exclusión social. (Kornfeld-Matte, 2020, párrafo 17).
En algunos países estas instituciones se encuentran en alerta máxima, muchos se han sometido a una cuarentena total, se les ha prohibido la entrada de visitantes, con la excepción de las situaciones del término de la vida, en otros se les permiten a las familias que lo deseen sacar a sus abuelos de las residencias de mayores, temporalmente y con derecho y garantías de no perder su plaza, siempre y cuando se debe mantener un aislamiento por seguridad, teniendo un carácter preventivo del contagio y humanitario al encontrarse con sus seres queridos.

\section{Cuidadores de adultos mayores que viven solos, poseen su asistencia 0 seguridad social}

Para este contexto resulta importante evaluar la figura de

[...] los cuidadores profesionales domiciliarios son los asignados por las instituciones de Seguridad y Asistencia Social, pudiendo ser externos o internos, y por último los cuidadores por beneficio contratados por el propio adulto mayor o por un familiar del mismo, que incluyen dos variantes: los contratados por un salario y los contratados por la obtención de bienes materiales, tales como: el disfrute de una vivienda, el uso de equipos electrodomésticos o medios de transporte, etc., esta última modalidad se utiliza con mucha frecuencia actualmente. (Díaz, 2016, p. 20).

Existe un incremento de "hogares unipersonales en este segmento de la población, lo que a su vez aumenta la probabilidad de aislamiento social y soledad no deseada. Las estrictas medidas de confinamiento suponen un gran reto que conllevan la ausencia o disminución de las interacciones sociales, lo que puede tener implicaciones para la salud física y mental". (Lara, 2020, párrafo 5).

\section{En Cuba con}

[...] la asistencia de los trabajadores sociales se investigó la situación de esas personas, con la propuesta de insertarlas en el Sistema de Atención a la Familia, y ayudarlos a higienizar sus hogares, existen más de mil unidades gastronómicas destinadas a brindar los alimentos a hogares de adultos mayores que viven solos y tienen dificultad para acudir a restaurantes, donde intervienen el Consejo de la Administración del Poder Popular, el Ministerio de Economía y Planificación, la Empresa de 
Comercio y Gastronomía, unido a líderes que identifican a estos grupos de poblaciones vulnerables. (Martínez, 2020, párrafo 6; Álvarez, 2020, párrafo 3).

Esta atención como experiencia cotidiana se caracteriza por su solidaridad y carácter humano, evidenciándose como fortaleza de nuestro sistema de salud en los momentos de eventualidad y aislamiento social en que nos encontramos ante la prevención de la pandemia.

\section{Adultos mayores totalmente desprotegidos}

Al evaluar esta contextualización, dudábamos, pues quienes serían los cuidadores de una persona que está totalmente desprotegida; y la respuesta es una, la misma persona que debería recibir la atención tiene cuidarse y cumplir el doble rol de sujeto-actor; resultaba difícil la búsqueda pues no es la realidad de nuestro país, sin embargo, sí la de muchos hogares, es por eso que mantuvimos el análisis en la clasificación.

Sólo el 30 por ciento de las personas mayores en el mundo están cubiertas actualmente por programas de pensión, y en la mayoría de las sociedades, la salud, el empleo y los servicios de transporte no son fácilmente disponibles o accesibles. Ante este comportamiento, podrían incrementarse las dificultades económicas y financieras de los adultos mayores. La gente que ha vivido con pobreza y desigualdad llega a la vejez con desventajas acumuladas. Su realidad es la de vivir solitarios afrontando el aislamiento social y enfrentando dificultades para realizar tareas cotidianas con las medidas adoptadas, encontrándose en un riesgo adicional los que no tienen familiares o amigos cercanos. (Stefanoni, 2013, párrafo 2; Huerta, 2020, párrafo 12).

Resulta significativo que,

[...] los prestadores de asistencia sanitaria y la sociedad en su conjunto presten atención a las necesidades especiales de los grupos de población de edad mayor mediante las medidas siguientes: capacitación de los profesionales sanitarios, prevención y atención de las enfermedades crónicas que acompañan a la vejez, elaboración de políticas sostenibles sobre la asistencia a largo plazo y los cuidados paliativos y creación de servicios y entornos que favorezcan a las personas de edad. (OMS, 2017 , párrafo 2).
La Organización Mundial de la Salud está trabajando para proporcionar las estrategias, soluciones y suministros cruciales que los países necesitarán. Todos podemos apoyar a los adultos mayores en estos tiempos. Muchos dependen de los servicios y apoyos que se les proporciona en el hogar o en la comunidad para mantener su salud e independencia. Si bien es cierto, que es importante el distanciamiento físico para evitar el contagio, también es importante comprender que no se trata de un aislamiento emocional con quienes o de quienes nos rodean. (Adhanom, 2020, párrafo 7; Orosa, 2020, párrafo 3).

Unido a lo anterior se presenta la victimización de los adultos mayores, teniendo como escenario la situación especial de la pandemia que atravesamos, siendo considerada desde nuestra óptica "como todo aquel maltrato o agresión física, psíquica, sexual y patrimonial que se desarrolla de forma habitual $o$ esporádica y trae como consecuencia un daño o una amenaza para la salud o la integridad del geronte". (Pérez, 2012, p. 51).

El análisis de este maltrato debe clasificarse a partir del estudio de tres elementos asociados a la acción dañosa: la voluntad del agresor, las causas que originan la agresión y las consecuencias que provoca. En la primera variable se evidencia la intencionalidad del abuso, el deseo o placer que desarrolla el victimario de hacer valer su autoridad o criterio mediante el acto despiadado; en el segundo elemento se representan las fuentes, procedencia y fundamentos en los que basan las agresiones, existiendo un número de factores psicológicos, sociales, económicos que se interrelacionan. El tercero se dirige a las consecuencias que provoca, en la mayoría sus secuelas provocan serios daños contra la salud, bienestar o integridad física. (Campillo, 2002, p. $6)$.

Sin pretender profundizar en los numerosos fenómenos asociados a los maltratos sobre este grupo etáreo, en estos momentos de confinamiento coincidimos que los factores de mayor incidencia son: el aislamiento social (separado de parientes y amistades), la situación de estrés del cuidador, la violencia generacional en el seno familiar, la violencia estructural, la violencia patrimonial (entendida no solo a las propiedades personales que posee sino también a la sustracción de los beneficios financieros recibidos).

La realidad ante la desprotección, el maltrato y el abandono, unido al envejecimiento poblacional y la vulnerabilidad que se incrementa, se une el 
reto de cuidarlos y protegerlos, para ello no solo debemos transformar los servicios asistenciales nos corresponde enfrentar el contexto con una mirada intencional e integradora y aunar fuerzas para frenar en lo posible que "el coronavirus cause más pobres, que muertos" (Ramonet, 2020, p. 45) en cualquier sociedad afectada.

\section{ELEMENTOS CONFIGURATIVOS DE LA FIGURA DEL CUIDADOR DEL ADULTO MAYOR}

Para adentrarnos al estudio del cuidador resulta vital analizar elementos que estructuran su representación, es por ello que desarrollaremos cada uno de ellos.

\section{Fundamentos de la labor del cuidador}

En este caso hacemos referencia a los cimientos, al sostén sobre el que recae esta tarea tan sacrificada, pero al igual tan digna de reconocimiento y de ejemplo. Consideramos que los pilares en que se sustenta el cuidado humano en la actualidad se sustenta en los motivos por el cual una persona decide asumir el cuidado de su semejante, siendo imprescindibles en los cuidados que se ofrecen, así como necesidades y derechos que surgen de los propios cuidados.

\section{Pero debemos valorar}

[...] la satisfacción del cuidador, donde a pesar de las dificultades y la dureza de la situación, los cuidadores manifiestan que cuidar a un familiar puede ser una de las experiencias más gratas y conmovedoras, porque a pesar de las numerosas tareas que ello implica, se puede descubrir condiciones o aptitudes que probablemente de otra forma hubieran pasado desapercibidas. (Díaz, 2016, p. 21).

\section{Motivación del cuidador}

El brindar cuidado es una labor que cualquiera no pudiera realizar, el cuidado trae consigo responsabilidades y cargas, pero no siempre esta labor es una decisión propia, en ocasiones no nos damos cuenta cuando estamos ofreciendo cuidados, pero poco a poco brindamos ayuda a otros para que puedan desarrollarse según las exigencias del entorno, hasta que un día reconocemos que sin nuestra ayuda no pueden convivir con el medio y es cuando tomamos la condición de cuidadores.
Somos del criterio que para ofrecer cuidados, tiene que existir un consentimiento propio, pues esta labor tiene que ir aparejada de sentimientos afectivos hacia la persona que se cuida, pero no siempre dichas emociones están presentes, "son diversas las razones por las que un sujeto decide cuidar de una persona que se encuentra en una situación de dependencia, entre ellos se encuentran: motivación altruista, reciprocidad, motivos económicos, sentimientos de culpa, evitar la censura del entorno y por obligación, abocada a ello por presiones familiares". (Montorio, 1999, p. 8).

La mayoría de las razones por la que se cuida a una persona mayor de edad llevan enraizados fuertemente los valores morales y de obligación. También se ha constatado que algunos de los cuidadores cuidan del adulto mayor para impedir la crítica de familiares o amigos que lo rechazarán por no cuidar de quien lo hizo con él.

Otras de las motivaciones son por necesidades económicas, mediante este empleo reciben una remuneración que no encuentran en otra alternativa laboral, o en otros casos viven agregados con familiares y el cuidar de otra persona en su domicilio le brinda techo y comida, aparte de un salario. En otras ocasiones el hilo que determina a una persona a cuidar a otra es un sentimiento de culpa, a partir de la existencia de dificultades o hechos en el pasado y se someten a cuidar al afectado en la actualidad buscando una compensación.

Como otra incitación podemos valorar los móviles altruistas o por reciprocidad, no en todos los casos los cuidadores tienen que tener un fin marcado, algunos conservan sentimientos de cariño, afecto, amor por la persona destinataria de los cuidados.

\section{Contenido elemental del cuidador}

Son múltiples las tareas que la persona en condición de cuidadora ofrece a otra que necesita de su ayuda para poder convivir con el entorno y poder satisfacer las necesidades cotidianas que la vida les exige, (Valderrama, 2020, párrafo 13), entre las labores más frecuentes que realizan los cuidadores se encuentran: ayuda en las actividades domésticas, control de sus medicinas, auxilio para el transporte fuera del domicilio, colaboración en tareas de enfermería, asistencia para la higiene personal, 
la alimentación establecida y los ejercicios que pueden desarrollar.

\section{El género y la feminización del cuidador}

Independientemente de la evolución y transformación en la familia patriarcal los hombres mantienen sus tradiciones $\mathrm{y}$ las mujeres a pesar del progreso alcanzado continúan asumiendo la responsabilidad del cuidado con una doble carga de trabajo.

"Los avances no han conseguido erradicar la discriminación ni han logrado evitar la pervivencia de un modelo androcéntrico, donde la transmisión de los roles de género es uno de los elementos que más contribuyen a perpetuar las desigualdades por medio de la socialización formal y no formal". (Mayobre y Vázquez, 2015, p. 84). En este sentido, llama la atención como perduran las figuras maternas en la ética del cuidado, mostrando la superioridad de las mujeres mediante una fundamentación biológica, justificando de esa forma la feminización del cuidado.

El esquema de cuidados avala la importancia del rol de hija cuidadora evidenciando

[...] tres variables que resultan fundamentales a la hora de determinar quién se tiene que ocupar de cuidar a la persona dependiente en el hogar: el sexo, el grado de parentesco y el estado civil. Así, ser mujer, hija o cónyuge, y, además, no tener pareja en ese momento, aúna mayores posibilidades de cuidar a la persona dependiente. (Mayobre y Vázquez, 2015, p. 92).

Hasta ahora las familias, y en ellas especialmente las mujeres, han sido las primeras actrices en el ejercicio de las tareas de bienestar (...). Por eso, en nuestros días, preguntarse por el futuro de los miembros más vulnerables de la sociedad requiere, no sólo analizar la crisis del Estado benefactor, sino, sobre todo, estudiar las consecuencias de tres cambios estructurales de envergadura: la incorporación de la mujer al mercado laboral, la transformación de la estructura familiar y la extinción de la mujer cuidadora (...). Y, por su parte, el Estado, carece de los recursos suficientes como para pagar unos trabajos de 24 horas, sin vacaciones, sin días de fiesta, que hasta ahora han hecho gratis las mujeres. (Cortina, 1999, p. 5).

En resumen,

[...] los rasgos más sobresalientes de esta realidad son la familiarización del cuidado y la feminización de las tareas. En conjunto el cuidado se mantiene con unos perfiles de género muy diferenciados marcados por la edad, las tareas a realizar, las implicaciones en la inserción profesional y las dimensiones afectivas. (...) La responsabilidad de los cuidadores es muy amplia, atendiendo necesidades que requieren una fuerte implicación, y que la intensidad del tiempo y del esfuerzo del cuidado es muy elevada. También se confirma la relevancia de la desigualdad de status (tanto por cuestión de género, clase u origen), ya que es precisamente en los niveles socioeconómicos inferiores en los que las mujeres se hacen cargo y se responsabilizan más de los dependientes, y es sobre ellas donde recae el mayor coste de tiempo y el mayor coste psicológico del cuidado. (Fernández, Artiaga y Dávila, 2013, p. 70).

\section{Necesidades de los cuidadores}

La variedad de tareas que deben asumir los cuidadores, la responsabilidad y compromiso que sobre ellos recae, trae como consecuencias que se vean alterados sus comportamientos habituales, como resultado de la dedicación que conlleva el cuidado de las personas que lo precisan, dejando al lado su propia vida para dar paso a la vida de la persona de quien se cuida.

\section{Las necesidades de los cuidadores}

[...] poseen un carácter multidimensional que transcurren desde una dimensión material en tanto es necesaria la comida, la vivienda, el abrigo y el agua, así como una inmaterial que refiere a afectos, relaciones sociales, libertad y autonomía. Estas dimensiones interactúan siempre, no existe separación, y adquieren el mismo valor. (...) Las necesidades implican recursos y derechos. En lo referente a cuidados, deben atenderse esas dos vertientes, en tanto ellos se pueden llevar a cabo, principalmente desde el trabajo no remunerado y forman parte de un proceso de feminización que se manifiesta en la incertidumbre de recursos económicos, escasas posibilidades laborales, exclusión de derechos sociales y prácticamente la dificultad de acceder a la autodeterminación de las mujeres. (Del Río, 2003, pp. 49-50).

Dentro de las necesidades que con más frecuencia presentan los cuidadores podemos encontrar el disponer de tiempo libre, lo cual se convierte en casi una utopía por lo que no hay espacio para el ocio, la recreación y el contacto con la sociedad, convirtiéndose esto en una insuficiencia vital, es por ello que se olvidan de sí mismos, dando paso al cuidado de otros. 
Otra insuficiencia que manifiestan los cuidadores, es el apoyo en el desarrollo de diferentes labores en el hogar, principalmente en las mujeres cuidadoras, porque sobre ella recaen las labores del hogar, lo que hace que se produzca una sobrecarga. También se encuentra el beneplácito que se reconozca la labor que desempeñan y no se le imponga, la familia debe ayudar a brindar cuidados y convertirse también en gestora de ellos. La falta de recursos económicos resulta ser otra demanda importante he insatisfecha ya que en oportunidades el cuidador deja su trabajo $o$ actividades generadoras de ingresos para poder cuidar de su familia y a su vez los gastos aumentan por las necesidades propias de la persona dependiente.

La dependencia afectiva y la variabilidad de necesidades del destinatario implica que las tareas que incluye el trabajo son en general difusas e imprecisas, y siempre deben concretarse en la relación interpersonal. Asimismo, la reivindicación de un trabajo digno y el cuestionamiento de la predisposición de las mujeres para los cuidados y su reparto entre hombres y mujeres, deben de ir acompañados, por un replanteamiento de los paradigmas normativos en torno a las necesidades sociales y quiénes deben hacerse cargo. (Fernández, Artiaga y Dávila, 2013, p. 58).

Resulta de gran importancia que los cuidadores puedan satisfacer sus necesidades y se vean apoyados por la familia, siendo esta la principal red de apoyo, es la encargada directa de brindar cuidados y de satisfacer demandas básicas, es muy fácil delegar labores ante la sensación de impotencia y desconcierto que se vive respecto al envejecimiento familiar, pero estas deberían ser tareas conjuntas, evitándose en el cuidador, el síndrome de burnout. (Buitrago, 2015, p. 39).

Con el apoyo e integración familiar no solo se beneficia el geronte, sino que disminuye la carga de responsabilidades del cuidador.

\section{Derechos de los cuidadores}

El brindar cuidado es una labor que exhibe mucho esmero y dedicación, lo que conduce a una difícil situación para aquellos individuos que lo ofrecen, llegando afectar su propia integridad, evaluemos entonces un conjunto de derechos a los que estos tienen acceso:

[...] dedicar tiempo y actividades sin sentimientos de culpa, experimentar sentimientos negativos por ver al enfermo o estar perdiendo a un ser querido, resolver aquello que seamos capaces y el derecho a preguntar sobre aquello que no se comprenda, buscar soluciones que se ajusten a sus necesidades y a de seres queridos, ser tratados con respeto por aquellos a quien se le solicita consejo y ayuda, y a seguir la propia vida. (Astudillo y Mendinueta, 2018, párrafos 6-7).

Teniendo en consideración el escenario en que se desarrollan los cuidadores, su espacio social y psicológico, resulta de gran apoyo los derechos anteriormente aludidos, constituyendo vías que posibilitan que se sientan aliviados con la carga emocional y laboral que llevan.

\section{PROTECCIÓN JURÍdICA EN CUBA AL CUIDADOR DEL ADULTO MAYOR}

A la par del envejecimiento de la población camina la figura del cuidador de los adultos mayores, de ahí la necesidad de evaluar jurídicamente la protección de sus derechos; con ese fin efectuamos un análisis en el ámbito del Derecho en Cuba. Abarcando la Constitución de la República, el Código de Trabajo y la Ley de Seguridad Social.

\section{La protección al cuidador en la Constitución de la República de Cuba}

Con el objetivo de realizar un análisis de la protección jurídica de los cuidadores, comenzamos por la Constitución de la República que precisa los principios generales de salvaguardia para la población, incluido el grupo etáreo que estamos evaluando. En el artículo 68 de la Constitución de la República de Cuba, "se aprecia que el Estado protege a los abuelos u otros familiares, en función del cuidado y atención". (Asamblea Nacional del Poder Popular en Cuba, 2019, p. 5).

Por su parte el artículo 84 de la Ley de leyes "aborda la obligación por parte de los hijos de respetar, atender y proteger sus madres, padres y otros parientes, lo que ayudará en su calidad y esperanza de vida". Mientras que el artículo 88 señala que,

[...] el Estado, la sociedad y las familias, en lo que a cada uno corresponde, tienen la obligación de proteger, asistir y facilitar las condiciones para satisfacer las necesidades y elevar la calidad de vida de las personas adultas mayores. De igual forma, respetar su autodeterminación, 
garantizar el ejercicio pleno de sus derechos y promover su integración y participación social. (ANPP, 2019, pp. 6-9).

Dado al nivel de envejeciendo de la población $\mathrm{y}$ por ende su proclividad a situaciones de riesgos resulta necesaria la inclusión del cuidador en el texto legal, apreciamos que aún no se identifica explícitamente como la figura que puede atender, cuidar y proteger a este grupo etáreo. Desde nuestra Carta Magna se otorga la responsabilidad de atender a los adultos mayores en dos aristas de análisis la familia y el Estado, sin incluir la realidad familiar de muchos contextos.

\section{La protección al cuidador en el Código de Trabajo}

El enfoque jurídico que establece el Código de Trabajo a los cuidadores para la protección de los adultos mayores, constituye un enorme reto ante el envejecimiento poblacional.

Solamente existe presencia de un artículo que aborda la atención a este grupo etáreo y lo hace "mediante las licencias no retribuidas a trabajadores con responsabilidades familiares, señalando el artículo 108 que, en el sector estatal a solicitud del trabajador, el jefe de la entidad puede conceder licencias no retribuidas a trabajadores con responsabilidades familiares para su atención y cuidado. (ANPP, 2013, p. 19).

Evidente resulta el insuficiente respaldo legislativo en la Ley laboral que establece las normativas que garantizan el amparo de los derechos y el respeto de los deberes, ante una necesidad creciente y real, lo cual entorpece el crecimiento de esta figura ocupacional.

\section{La protección al cuidador en la Ley de Seguridad Social}

En nuestro país se encuentran vigente la Ley No. 105/08 de Seguridad Social y su Reglamento, el Decreto Ley 203/08, (ANPP, 2008) ambos cuerpos legales mantienen el reconocimiento de la asistencia social a domicilio, servicio que persigue brindar apoyos complementarios, de carácter personal y social, en el domicilio de personas que vivan solas y poseen impedimentos para el desarrollo cotidiano. De esta manera, se logra que los gerontes imposibilitados de realizar las diligencias diarias de forma autónoma; reciban protección en su propio medio, satisfagan sus principales necesidades biológicas, psicológicas, comunicativas y sociales.

El asistente social a domicilio, también llamado cuidador, desempeña diversas funciones, las cuales no han sido normadas, pero si definidas en una metodología del Ministerio de Trabajo y Seguridad Social que indica los procedimientos para el otorgamiento, ejecución y revisión de las prestaciones de la asistencia social.

Son incuestionables los resultados logrados en el área de seguridad y asistencia social, en consecuencia, los beneficios que por estos conceptos reconoce el ordenamiento jurídico se han ido modificando, valorando en cada caso, el servicio de asistencia social a domicilio no ha estado exento de ello, pese a las dificultades existentes en su concepción, como las cuestiones sobre las funciones del cuidador y el control de su cumplimiento.

En principio, resulta comprensible que el Estado no debe cargar al presupuesto de la asistencia social el cuidado de aquellas personas que, por razón de la edad, dado el grado de dependencia que poseen, se encuentran requeridos de un asistente social a domicilio teniendo un familiar que le corresponde asumir dicha responsabilidad.

De lo anterior se deduce la imperante realidad de un gran número de cuidadores informales o no oficiales, que ofrecen cuidados a personas adultas dependientes y no poseen un respaldo legal por lo que es conveniente formalizar la situación de los cuidadores no designados por el servicio de asistencia social y dotarlos de beneficios, atenciones y garantías como consecuencia de su carga, pues tanto los formales como los informales desarrollan la misma actividad.

Tomando como base la idea anteriormente abordada surge la necesidad de la inclusión de los cuidadores informales en nuestras normas jurídicas con el objetivo de formalizar y recoger legalmente su estatus social, situación que le concede un conjunto de garantías actualmente no previstas en ningún ordenamiento jurídico.

Bajo el prisma de las Ciencias Jurídicas se abre un abanico de interrogantes relacionadas con la necesaria corresponsabilidad del Estado y la familia en el cuidado de este grupo etáreo, máxime de aquellos que presentan un alto grado de vulnerabilidad, ya sea porque viven 
solos, padecen de enfermedades que aceleran el deterioro físico-mental, han adquirido con la edad alguna discapacidad, reciben poco o ningún apoyo familiar o se encuentran en estado de abandono. (León, 2020, p. 65).

La atención y cuidados ante el envejecimiento poblacional es un hecho que sin duda se ha convertido en una problemática tanto social como jurídica, situación que nos obliga de forma inmediata a buscar vías y estrategias integrales para su solución.

\section{CONCLUSIONES}

El estudio doctrinal de la figura del cuidador evidencia la existencia de diversas definiciones conceptuales y teóricas. La definición que se propone se ajusta a considerarlo desde dos ópticas diferentes, el cuidado informal fundado en una correlación social basada en afecto y cariño que se realiza mediante una relación de parentesco, vecindad o amistad; y el cuidado formal ejecutado por asistentes, personal de enfermería, entre otras.

La pandemia COVID-19 ha demostrado la vulnerabilidad de los adultos mayores de agravarse al contraer la enfermedad; la mayoría de los Estados como parte del propio proceso de enfrentamiento no implementaron oportunas políticas de prevención de riesgos ni acciones integrales de sus estructuras para afrontar y reducir este flagelo; en el caso específico de Cuba se aprobó un plan de acciones que tiene su basamento en la contención del virus, la disminución de las secuelas perjudiciales para la salud, con énfasis en este grupo de riesgo y su impacto en la esfera económica-social, demostrando cada día la coherencia de su estrategia de enfrentamiento.

En la estructuración de las clasificaciones de los cuidadores del adulto mayor de acuerdo al ambiente o contexto de ocurrencia, se reconoce la presencia de cuatro juicios organizativos de análisis: cuidadores para adultos mayores que viven en el seno de la familia; cuidadores para adultos mayores a cargo del Estado; cuidadores para adultos mayores que viven solos, pero poseen su asistencia o seguridad social; y adultos mayores totalmente desprotegidos.

El cuidador del geronte necesita ser potenciado desde un enfoque interdisciplinario que contenga, tanto la regulación informal como la regulación formal. El análisis informal se sostiene en un enfoque victimocriminológico, aspecto que conlleva la mirada de otras ciencias como la sociología, la medicina, la psicología social, entre otras; mientras la regulación formal se conforma en las disímiles perspectivas jurídicas de amparo de esta figura. En Cuba la variante formal evidenciada en la protección jurídica nos muestra que no existe un reconocimiento legislativo al cuidador, ni mecanismos institucionales implementados.

\section{BIBLIOGRAFÍA}

Adhanom Ghebreyesus, T. (2020). Alocución de apertura del Director General de la OMS ?. Recuperado de https://www.who.int/es/ $\mathrm{dg} /$ speeches/detail/who-director-general-sopening-remarks-at-the-edia-briefing-oncovid-19.

Álvarez Palacio, D. (23 de abril de 2020). Ante la COVID-19: Atención al adulto mayor en una zona en cuarentena. En Periódico Granma, Recuperado de http:// www.granma.cu/fotorreportajes/2020-04-23/ an te-1a-covid-19-solidaridad-yhumanismo-23-04-2020-18-04-18.

Alvero Francés, F. ( $3^{a}$ ed). (1978). Diccionario Cervantes Manual de la Lengua Española, Cuba, Editorial Pueblo y Educación.

Anaya Cruz, B. (2020). Coronavirus, sistema de salud y economía en Cuba. Recuperado de https://www.cubainformacion.tv/ cuba/20200403/85371/85371-coronavirussistema-de-salud-y-economia-en-cuba.

Astudillo, W. y Mendinueta, C. (2018). Derechos de los enfermos y cuidadores. Recuperado de http://www.indesol.gob.mx/ web/Index.php? $1=12$.

Barrios, M. et al. (22 de marzo 2020). Mi Hogar, mi refugio. En Periódico digital Juventud Rebelde, Recuperado de http://www. juventudrebelde.cu/cuba/2020-03-22/mihogar-mi-refugio.

Buitrago Echeverri, M. T. (2015). Necesidades de cuidados que tienen los cuidadores de personas en situación de discapacidad por parte de su familia en la localidad de Fontibón. (Tesis para optar por el título de Enfermería), Pontificia Universidad Javeriana, Colombia. 
Campillo Motiva, R. (2002). Violencia con el anciano. En: Revista Cubana de Medicina General Integral, No. 18 (4), pp., 2-10, Cuba.

Cortina, A. (22 de noviembre de 1999). La extinción de la mujer cuidadora. Periódico El País, España, p. 5.

Cruz Rivas Herrera, J. y Ostiguin Meléndez, R. M. (2016). Cuidador: ¿concepto operativo o preludio teórico? Revista Enfermería Universitaria, 8 (1), México, pp. 49-54.

De La Cuesta Benjumeda, C. (2013). ( $3^{\mathrm{a}}$ ed). El cuidado del otro: Desafíos y posibilidades, España, Editorial Alianza.

De Los Reyes, MC. (2008). Construyendo el concepto del cuidador de ancianos, Foro de Investigación, Envejecimiento de la población en MERCOSUR, Curitiba, Brasil.

Del Río, S. (2003). La crisis de los cuidados: precariedad a flor de piel. Rescoldos. Revista de Diálogo Social, No. 9, España, pp. 47-57.

Devandas, C. (2020). Es inaceptable segregar a una persona por ser mayor y tener una discapacidad. Red latinoamericana de Gerontología. Recuperado de https:// www.gerontologia.org/portal/information/ showInformation.php?idinfo $=4405$.

Díaz Echemendía, L. (2016). El tratamiento jurídico a la figura del cuidador del adulto mayor en Cuba. (Trabajo de diploma en opción al título de Licenciado en Derecho), Universidad de Ciego de Ávila Máximo Gómez Báez, Cuba.

Fernández Moreno, S. A. (2 de abril 2020). Prevenimos Problemas o ayudamos a solucionarlos. Sitio Social Laboral Legal Today. Recuperado de http://www.legaltoday. com/practica-juridica/social-laboral/ prevencion_riesgos_laborales/referencias-alcovid-19.

Fernández Villanueva, C., Artiaga Leiras, A. y Dávila de León, M. C. (2013). Cuidados, género y transformaciones de identidades. En: Cuadernos de Relaciones Laborales. Volumen 31(1), España, pp. 57-89.

Gallardo, P. y Rojas, M. (2016). El rol del cuidador en el adulto mayor. (Investigación de la Escuela de Enfermería), Facultad de
Ciencias Médicas, Universidad Nacional de Cuyo, Argentina.

Guimaráes, M. (2015). Aspectos psicológicos en cuidadores formales, (Tesis en opción al grado científico de Doctor en Ciencias de la Salud), Universidad de Madrid, España.

Hadar, Y. (20 marzo 2020). El número de pobres en la América Latina puede crecer en 35 millones por el coronavirus COVID-19. Noticias ONU, Asuntos Económicos, Recuperado de https://news.un.org/es/ story/2020/03/1471522.

Huerta Mendoza, 1. (30 de marzo 2020). Consecuencias de aislar a los ancianos durante la pandemia de COVID-19, Gaceta UNAM, Recuperado de https://www.gaceta.unam.mx/ aislar-a-los-ancianos-durante-la-pandemiareduciria-la-transmision-de-covid-19/.

Kornfeld-Matte, R. (2020). Es inaceptable el abandono de personas mayores en los asilos durante la pandemia. Recuperado de http://www.radiobayamo.icrt.cu/2020/03/28/ es-inaceptable-el-abandono-de-personasmayores-en-los-asilos-durante-la-pandemiadel-coronavirus/.

Lara Pérez, E. (2020). COVID-19: Cómo ayudar a las personas mayores que viven solas. Recuperado de https://theconversation. $\mathrm{com} /$ covid-19-como-ayudar-a-las-personasmayores-que-viven-solas-133663.

León García, L. (2010). La pluralidad del régimen tuitivo de los adultos mayores ante el deterioro de la capacidad fisica y mental. (Tesis presentada en opción al título de especialista en Derecho Civil y Patrimonial de Familia), Universidad Central Marta Abreu de las Villas, Cuba.

Martínez Zelada, L. M. (25 marzo 2020). Prioriza comercio atención a adultos mayores solos, ante pandemia, Agencia cubana de noticias, Recuperado de http://www.acn.cu/ cuba/62490-prioriza-comercio-atencion-aadultos-mayores-solos-ante-pandemia.

Mayobre, P. y Vázquez, I. (2015) Cuidar cuesta: un análisis del cuidado desde la perspectiva de género. Revista Española de Investigaciones Sociológicas, No 151, España, pp. 83-100. 
Ministerio de Salud Pública de la República de Cuba. (2020). Para prevenir y enfrentar el nuevo coronavirus, participación popular es esencial. Recuperado de https://salud.msp.gob. $\mathrm{cu} / \mathrm{p}=4023$.

Ministerio de Salud Pública de la República de Cuba. (5 de mayo 2020). Parte de Cierre del 4 de mayo 2020. Recuperado de https://salud. msp.gob.cu/.

Montorio Cerrata, I, Fernández de Trocóniz, M.I. y Díaz Veiga, P. (1999). Cuando las personas mayores necesitan ayuda, Revista Guía para cuidadores y familiares, 3 (1), Ministerio de Asuntos Sociales, España, pp. 229-248.

Navarrete Calderón, C. (2004). Criminología: Alcances, Ciencia, Disciplina y Práctica Social, Colectivo de Autores, Texto Criminología, Capítulo I, Cuba, Editorial Félix Varela.

Oficina Nacional de Estadísticas e Información. (2018). Anuario Estadístico, Población. Cuba.

Organización Mundial de la Salud. (5 de mayo 2020). En Coronavirus disease 2019 (COVID-19) Situation Report-101. World Health Organization. Infection prevention and control during health care when COVID-19 is suspected.

Organización Mundial de la salud. (2017). La salud mental y los adultos mayores. Recuperado de https://www.who.int/es/newsroom/fact-sheets/detail/la-salud-mental-y-losadultos-mayores. Organización Mundial de la Salud. (2018). Informe Mundial sobre la violencia y la salud.

Orosa Fraíz, T. (28 marzo 2020). Las personas mayores ante la COVID-19. En Cubadebate, Recuperado de http://www.cubadebate.cu/ especiales/2020/03/28/las-personas-mayoresante-la-covid-19/\#.Xq4oxHJuncc.

Pérez del Molino Martín, I. y Moya López, M. (3 $3^{\mathrm{a}}$ ed). (2000). Cuidadores de ancianos profesionales, Manual para el Cuidado de ancianos, Argentina.

Pérez Cabrera, F. (2a ed). (2016). Cuidadores de Ancianos Profesionales, Cuba, Editorial Pueblo y Educación.
Pérez Nájera, C. (2012). Violencia sobre el adulto mayor. Estrategia para reducir la victimización en el municipio de Ciego de Ávila. (Tesis en opción al Grado de Doctor en Ciencias Jurídicas). Universidad de la Habana, Cuba.

Portal Miranda., J. A. (2020). Reorganiza Cuba su sistema de salud para hacer más efectiva la lucha contra el coronavirus. Recuperado de http://www.radiohc.cu/noticias/salud/217731reorganiza-cuba-su-sistema-de-salud-parahacer-mas-efectiva-la-lucha-contra-elcoronavirus.

Ramonet, I. (2020). Ante lo desconocido. La Pandemia y el sistema-mundo. En Cubadebate, Recuperado de http://www.cubadebate.cu/ especiales/2020/04/25/especial-de-ignacioramonet-ante-lo-desconocido-la-pandemia-yel-sistema-mundo/.

Stefanoni, S., (2013). Los mejores (y los peores) países para ser viejo. Recuperado de https://www.bbc.com/mundo/ noticias/2013/10/130726_salud_indice paises_vejez_men.

Valderrama, H. (2019). Cuidadores de ancianos profesionales. En: Cursos de Formación Multimedial, Recuperado de https://www. cuidadoresdeancianos.com/cuidadores/cuidadoresprofesionales/.

\section{LEGISLACIONES}

Asamblea Nacional del Poder Popular, (2019). Constitución de la República de Cuba, Dirección de Legislación y Asesoría del Ministerio de Justicia, Cuba.

Asamblea Nacional del Poder Popular, (2013). Ley No. 116 del 20 de diciembre, Gaceta Oficial No. 29 Extraordinaria de 17 de junio de 2014, Cuba.

Asamblea Nacional del Poder Popular, (2008). Ley No. 105, Ley de Seguridad Social y su Reglamento, 27 de diciembre, Cuba. 